\title{
Head and Neck Neuroendocrine
} Carcinoma

National Cancer Institute

\section{Source}

National Cancer Institute. Head and Neck Neuroendocrine Carcinoma. NCI Thesaurus.

Code C160980.

A neuroendocrine carcinoma that arises from the head and neck. 